\title{
Behaviour of large-area avalanche photodiodes under intense magnetic fields for VUV- visible- and X-ray photon detection
}

\author{
L.M.P. Fernandes ${ }^{\mathrm{a}}$, A. Antognini ${ }^{\mathrm{b}}$, M. Boucher ${ }^{\mathrm{c}}$, C.A.N. Conde ${ }^{\mathrm{a}}$, O. Huot ${ }^{\mathrm{c}}$, \\ P. Knowles ${ }^{c}$, F. Kottmann ${ }^{\text {d }}$, L. Ludhova ${ }^{c}$, F. Mulhauser ${ }^{c}$, R. Pohl ${ }^{\text {be }}$, \\ L.A. Schaller ${ }^{\text {c }}$, J.M.F. dos Santos ${ }^{\mathrm{a}, *}$, D. Taqqu ${ }^{\mathrm{e}}$, J.F.C.A. Veloso ${ }^{\mathrm{a}}$

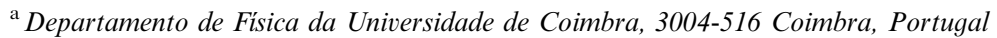 \\ ${ }^{\mathrm{b}}$ MaX-Planck-Institut für Quantenoptik, DE-85748 Garching, Germany \\ ${ }^{\mathrm{c}}$ Departement de Physique de l'Université, CH-1700 Fribourg, Switzerland \\ ${ }^{\mathrm{d}}$ Institut für Teilchenphysik, ETHZ, CH-8093 Zürich, Switzerland \\ ${ }^{\text {e }}$ Paul Scherrer Institut, CH-5232 Villigen-PSI, Switzerland
}

Received 19 April 2002; received in revised form 29 October 2002; accepted 18 November 2002

\begin{abstract}
The behaviour of large-area avalanche photodiodes for X-rays, visible and vacuum-ultra-violet (VUV) light detection in magnetic fields up to $5 \mathrm{~T}$ is described. For X-rays and visible light detection, the photodiode pulse amplitude and energy resolution were unaffected from 0 to $5 \mathrm{~T}$, demonstrating the insensitivity of this type of detector to strong magnetic fields. For VUV light detection, however, the photodiode relative pulse amplitude decreases with increasing magnetic field intensity reaching a reduction of about $24 \%$ at $5 \mathrm{~T}$, and the energy resolution degrades noticeably with increasing magnetic field.
\end{abstract}

(C) 2002 Elsevier Science B.V. All rights reserved.

PACS: $07.85 . \mathrm{F} ; 29.40 . \mathrm{M} ; 85.60 . \mathrm{D}$

Keywords: Photon detectors; X-ray detection; Avalanche photodiodes; Magnetic field

\section{Introduction}

Recent advances on the development of largearea avalanche photodiodes (LAAPDs) with higher gains and improved spatial uniformity have prompted intensive studies of their characteristics for scintillation detection. LAAPDs have been

*Corresponding author. Tel.: + +351-239410667; fax: +351 239829158..

E-mail address: jmf@gian.fis.uc.pt (J.M.F. dos Santos). mostly used as optical photodetectors coupled to inorganic scintillators for $\mathrm{X}$ - and $\gamma$-ray detection, substituting for photomultiplier tubes. Applications include instrumentation for nuclear physics $[1,2]$, high-energy physics [3, 4] and medicine $[5,6]$. Additionally, these devices are fairly good $\mathrm{X}$-ray spectrometers $[7,8]$ presenting energy resolutions similar to those of proportional counters [8].

More recently, windowless LAAPDs with sensitivity extended to the vacuum-ultra-violet (VUV) region became commercially available and their 
application as VUV photonsensors for the scintillation light from noble gases and liquids in positron-emission tomography, $X$ - and $\gamma$-ray spectroscopy is under investigation [9-11].

The use of LAAPDs in an experiment to measure the Lamb-shift of the $2 \mathrm{~S}-2 \mathrm{P}$ atomic states in muonic hydrogen $(\mu \mathrm{p})$ is being considered [12]. The experiment, to be carried out at the Paul Scherrer Institute (PSI), Switzerland, in collaboration with nine institutions, consists in obtaining long-lived $\mu$ p atoms in the 2S-metastable state by stopping a low energy $\mu^{-}$beam in a small volume of low-pressure hydrogen in a $5 \mathrm{~T}$ magnetic field. A pulsed beam from a tuneable laser induces the $2 \mathrm{~S}$ $2 \mathrm{P}$ transition in $\mu \mathrm{p}$ and the $1.9-\mathrm{keV} \mathrm{X}$-ray photons resulting from the $2 \mathrm{P}-1 \mathrm{~S}$ de-excitation will be detected. Measuring the coincidences between the laser pulse and the $1.9-\mathrm{keV} \mathrm{X}$-rays as a function of the laser wavelength, the Lamb shift can be determined. Low counting rates are expected and the 1.9-keV X-ray background will be reduced by gating its coincidence with the signal resulting from the high-energy electron produced by the subsequent muon decay. The X-ray detector should be compact and insensitive to a $5 \mathrm{~T}$ magnetic field.

The LAAPD can be used in two detector configurations: as the VUV photosensor of a xenon gas proportional scintillation counter (GPSC) [13] or as a direct X-ray detector [8]. The superior signal-to-noise ratio, large-area capability and energy resolution of a GPSC are advantages. However, the compactness and windowless design, the simple operation and the use of much lower biasing high-voltage, for the LAAPD operation as a direct $\mathrm{X}$-ray detector configuration, present important advantages in this application. In both cases, the LAAPD needs to be operated under intense magnetic fields, up to $5 \mathrm{~T}$.

The very small effect of magnetic field on the operation of the avalanche photodiodes has often been referred to in the literature [3, 4, 14]. However, detailed experimental results on this issue are scarce. Also, the experimental results available from manufacturers have not been published, to the best of our knowledge. Additionally, most of the studies with LAAPDs were carried out for visible light leaving aside the VUV and X-ray range. It has been proved that some LAAPD characteristics are different for visibleand VUV-light detection $[15,16]$.

In this work we present an experimental study on the behaviour of LAAPDs under magnetic fields up to $5 \mathrm{~T}$ for X-ray, VUV- and visible-light detection. LAAPDs were used to detect directly 5.4-keV X-rays, to read the scintillation light of a xenon GPSC and the scintillation light of a CsI (Tl) scintillation crystal. Detectors mean pulse amplitudes and energy resolutions were monitored as a function of the magnetic field. The experiment was performed in a cryogenic superconducting solenoid at PSI [17].

\section{Experimental set-up}

Avalanche photodiodes are compact, monolithic devices made of a silicon $\mathrm{p}-\mathrm{n}$ junction where the internal electric field can reach values high enough to allow electron multiplication by impact ionisation $[14,18,19]$. When a high voltage is applied to the photodiode only a small region of the p-layer in the front part of the diode remains undepleted - the drift region (Fig. 1). The electric field in this region is low but in the depleted region increases with the depth, presenting a maximum around the $\mathrm{p}-\mathrm{n}$ junction. An incident photon, absorbed in the drift region or in the p-depleted layer, produces electron-hole pairs and the resulting electrons are accelerated towards the $\mathrm{n}+$ contact, undergoing avalanche multiplication due to the high electric field around the junction. Charge gains of a few 100 are typical, depending exponentially on the applied voltage.

The different detector configurations used in this work are presented in Fig. 2. In all cases, LAAPDs $16-\mathrm{mm}$ in diameter, ${ }^{1}$ biased with $1800 \mathrm{~V}$, were used. For each run, ambient temperature inside the superconducting bore remained at about $20^{\circ} \mathrm{C}$ within $\pm 1^{\circ} \mathrm{C}$ and amplitude corrections of $5.4 \%$ per ${ }^{\circ} \mathrm{C}$ were carried out [16]. The thickness of the LAAPD drift region is about $10 \mu \mathrm{m}$, while the charge-multiplication region begins at a depth of

\footnotetext{
${ }^{1}$ Advanced Photonix, Inc., 1240 Avenida Acaso, Camarillo, CA 93012, USA.
} 
about $20 \mu \mathrm{m}$ in the depleted region and has a thickness of about $20 \mu \mathrm{m}$ [14].

For direct detection of X-rays with LAAPD an $\mathrm{X}$-ray beam from a ${ }^{54} \mathrm{Mn}$ radioactive source was used (Fig. 2a). The X-ray interacts directly in the $\mathrm{Si}$ and the resulting primary electrons are amplified by the intense electric field around the junction producing a pulse with amplitude that is, in average, proportional to the X-ray energy.

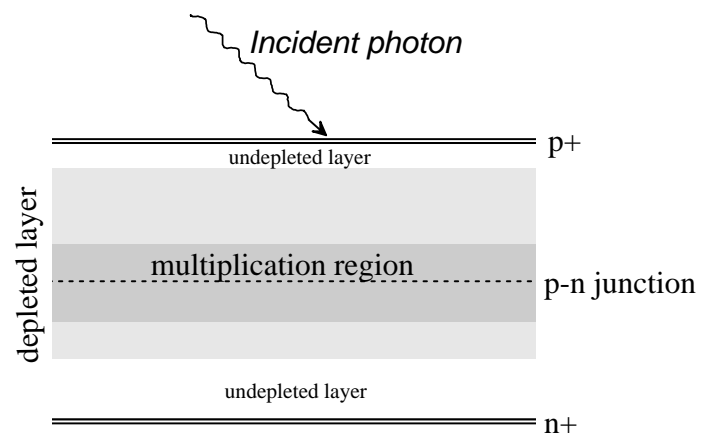

Fig. 1. Schematic diagram of a typical avalanche photodiode section.
For visible-light detection, $835-\mathrm{keV} \quad \gamma$-rays emitted from the ${ }^{54} \mathrm{Mn}$ radioactive source were allowed to interact in a $1 \times 1 \times 1-\mathrm{cm}^{3}$ CsI (Tl) scintillation crystal, placed above the LAAPD used to readout the scintillation (wavelength around $520 \mathrm{~nm}$ ) produced by each $\gamma$-ray interaction (Fig. 2b). The pulse amplitude at the LAAPD output is proportional to the amount of detected scintillation and, thus, in average to the $\gamma$-ray energy.

For VUV-light detection the LAAPD was placed inside the gas envelope of a driftless GPSC [13], which has a $1.1-\mathrm{cm}$ thick scintillation region filled with xenon at 1140 mbar (Fig. 2c). The gas volume is sealed and uses a small getter for gas purification (SAES St 172). The 12.5- $\mu$ m-thick, 10$\mathrm{mm}$-diameter aluminised Mylar window is maintained at $-6 \mathrm{kV}$. A Macor ceramic insulates the window holder from the detector body. The LAAPD was positioned just below the anode grid $(80-\mu \mathrm{m}$-diameter stainless steel wire with $900-\mu \mathrm{m}$ spacing). The anode grid, the photosensor body, and the detector body were maintained at ground
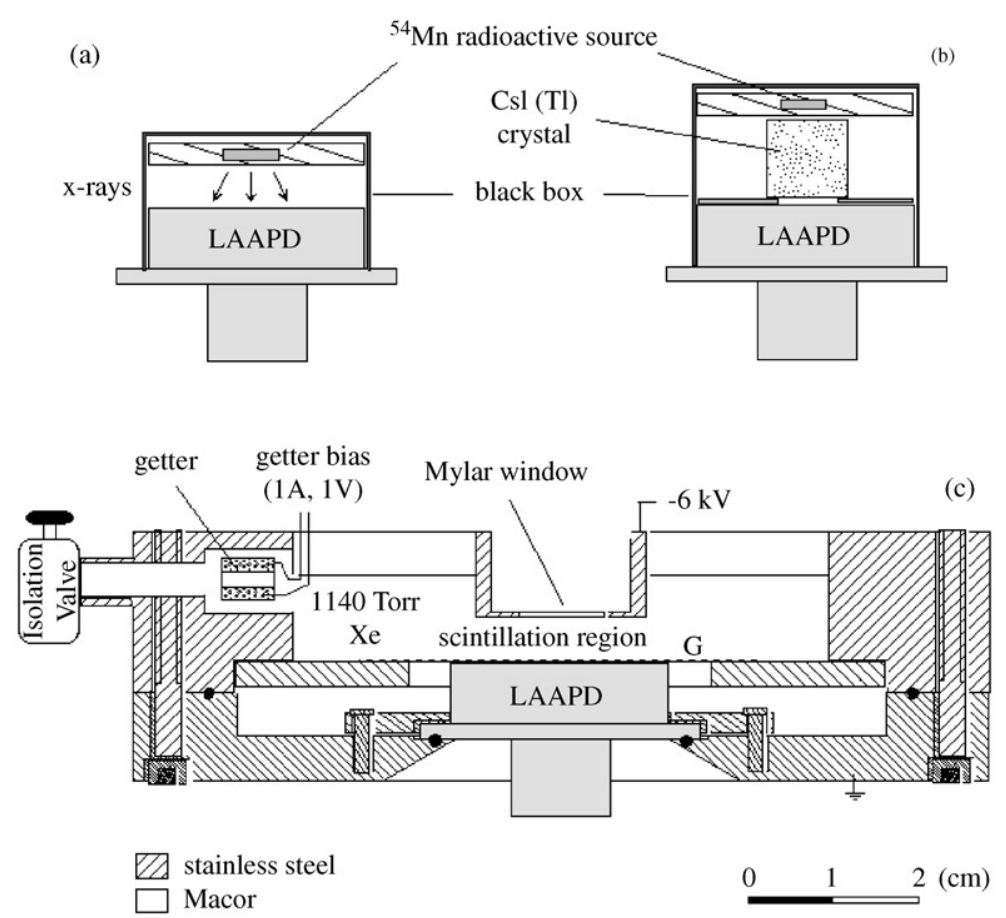

Fig. 2. Different detector set-ups for LAAPD-detection of X-rays (a), visible-light (b) and VUV-light (c). 
potential. The Macor, the radiation window and holder and the detector body were vacuum-sealed using a low-vapour pressure epoxy, while the photosensor body was vacuum-sealed to the detector body by compressing an indium gasket. A 2-mm collimated X-ray beam from a ${ }^{55} \mathrm{Fe}$ radioactive source was allowed to interact in the xenon. The electric field inside the detector is sufficiently high for the primary electrons resulting from each X-ray interactions to excite but not ionise the gas atoms along their path towards the anode grid. In the de-excitation processes VUV scintillation photons (wavelength around $172 \mathrm{~nm}$ ) are emitted and the average amount of scintillation light detected by the LAAPD is proportional to the X-ray energy.

The detectors were installed in a black box to shield from the ambient light. The LAAPD signals were fed through a low-noise charge preamplifier (Canberra 2004, with a sensitivity of $45 \mathrm{mV} / \mathrm{MeV}$ ) to a spectroscopy amplifier and were pulse-height analysed by a multi-channel analyser. Shaping time-constants of $0.2,1$ and $2 \mu$ s were used in the main amplifier for X-ray, visible and VUV detection, respectively: $0.2 \mu \mathrm{s}$ is optimum for X-ray pulses in the LAAPD; $1 \mu$ s corresponds to the time characteristic of the $\mathrm{CsI}(\mathrm{Tl})$ scintillation; $2 \mu$ s corresponds to the electron drift time in the xenon gas. For pulseamplitude and energy resolution measurements the pulse-height distributions are fitted to a Gaussian function superimposed on a linear background, from which the centroid and the full-width at halfmaximum (FWHM) are determined.

Each detector system was placed in a 1-m-long and 20-cm-bore-diameter superconducting solenoid capable of achieving magnetic fields up to $5 \mathrm{~T}$, uniform $\left(10^{-4}\right)$ over an axial distance of $30 \mathrm{~cm}$ from the centre. The amount of scintillation light collected in the LAAPD was kept constant during the experiment for both visible and VUV cases. Detector pulse amplitude and energy resolution were monitored as a function of the magnetic field, varying the magnetic field intensity inside the solenoid in $1 \mathrm{~T}$ steps up to $5 \mathrm{~T}$. The detector orientation inside the solenoid was chosen such that the LAAPD axis was perpendicular to the magnetic field direction, the most unfavourable orientation, Fig. 3.

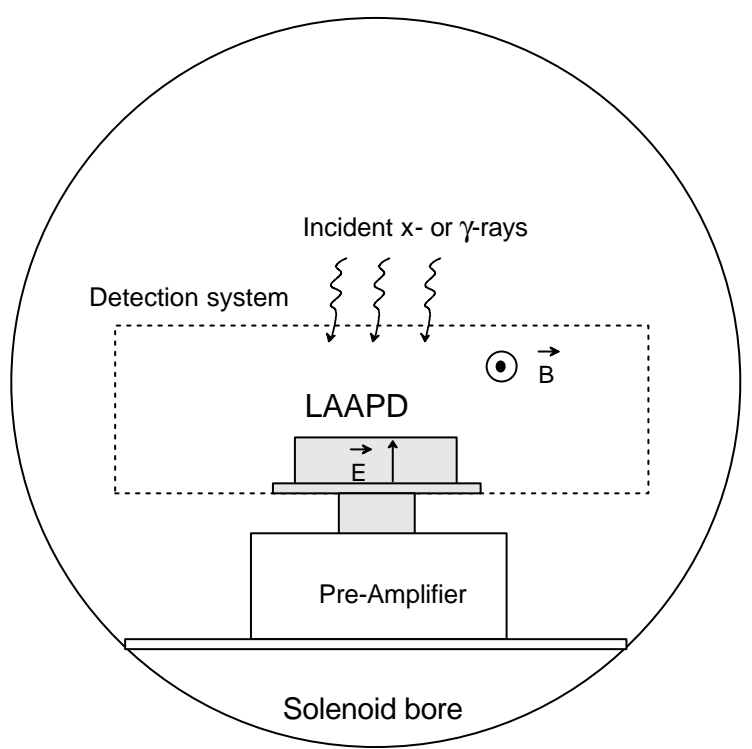

Fig. 3. Schematic of the detector/LAAPD orientation relative to the electric and magnetic fields in the experimental set-up.

\section{Experimental results and discussion}

In Fig. 4 we present pulse-height distributions obtained for each case, and for magnetic fields of 0 and $5 \mathrm{~T}$. For the X-ray detection cases (Figs. 4a and $4 \mathrm{c}$ ), spectral features include the $\mathrm{Cr}$ or the $\mathrm{Mn}$ $\mathrm{K}$-lines, the respective escape peaks and the tail due to the electronic noise in the low-energy limit. For the $\gamma$-ray detection (Fig. 4b) the pulse-height distribution depicts the peak corresponding to the full-energy absorption, the Compton continuum and the electronic noise tail. As shown in Fig. 4, there is no significant degradation of the obtained pulse-height distributions with the magnetic field intensity for the cases of X-rays and visible light detection with the LAAPD. On the other hand, for the VUV-scintillation detection, pulse amplitude reduction above $20 \%$ can be observed. In all cases, the electronic noise tail in the low-energy limit did not change with the magnetic field.

Figs. 5a and $\mathrm{b}$ present the detector relative pulse amplitude and energy resolution, respectively, as a function of the magnetic field intensity for the three different cases. For X-ray and visible-light detection with the LAAPD, amplitude variations are less than $1.5 \%$, being within the experimental 

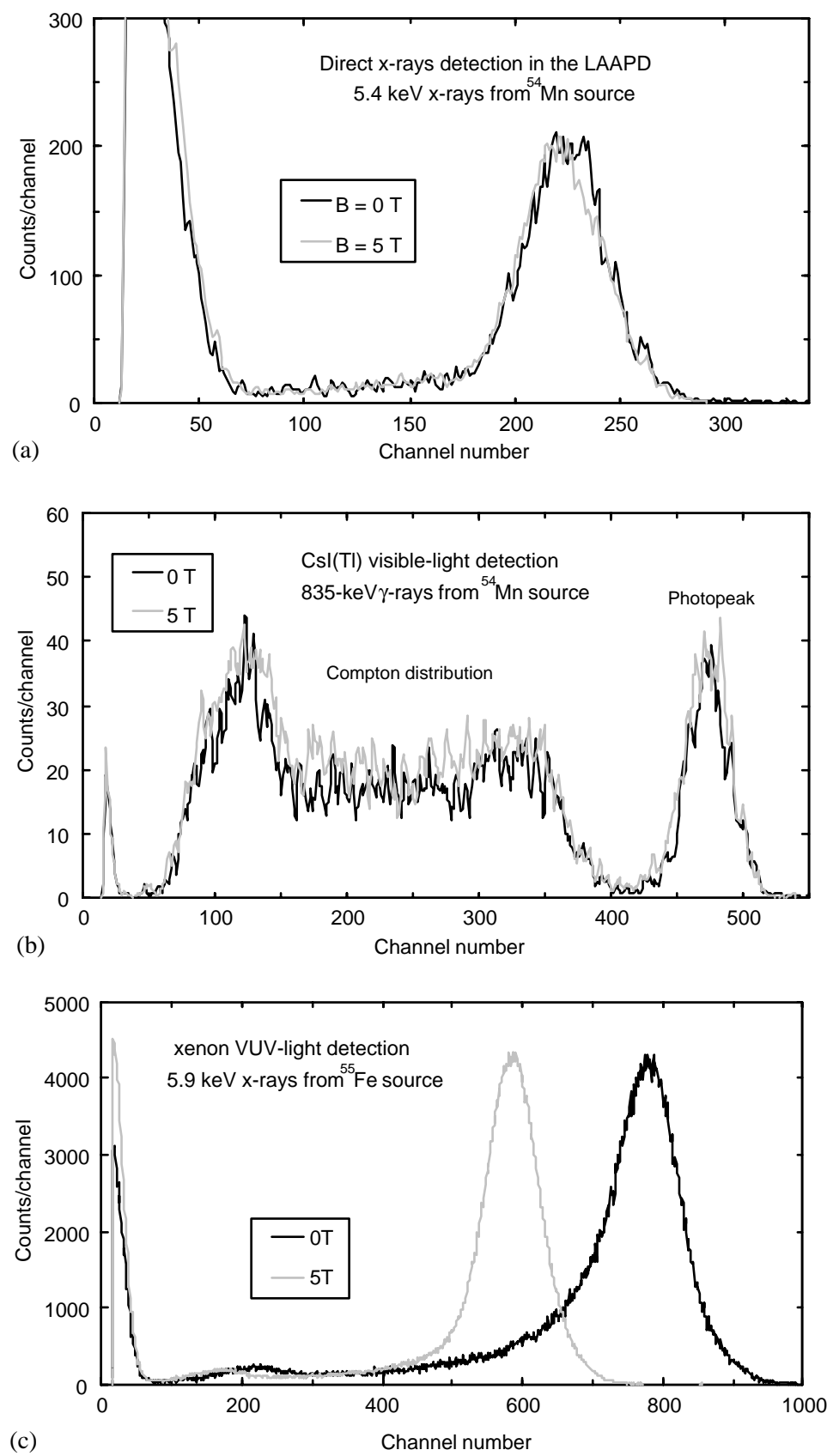

Fig. 4. Pulse-height distributions obtained with the different detector set-ups. (a) direct $\mathrm{Cr} \mathrm{K}_{\alpha, \beta} \mathrm{X}$-ray detection in the LAAPD, (b) $835-\mathrm{keV} \gamma$-rays interacting in a $\mathrm{CsI}(\mathrm{Tl})$ crystal and (c) $\mathrm{Mn} \mathrm{K}_{\alpha, \beta} \mathrm{X}$-rays interacting in a Xe-GPSC, using the LAAPD for the visible- and VUV-scintillation detection, respectively.

error. Also, no significant variations of the energy resolution are observed for these two cases. For the VUV-light detection with the LAAPD sig- nificant variations are noticeable: the relative pulse amplitude decreases gradually with increasing magnetic field intensity reaching a reduction of 

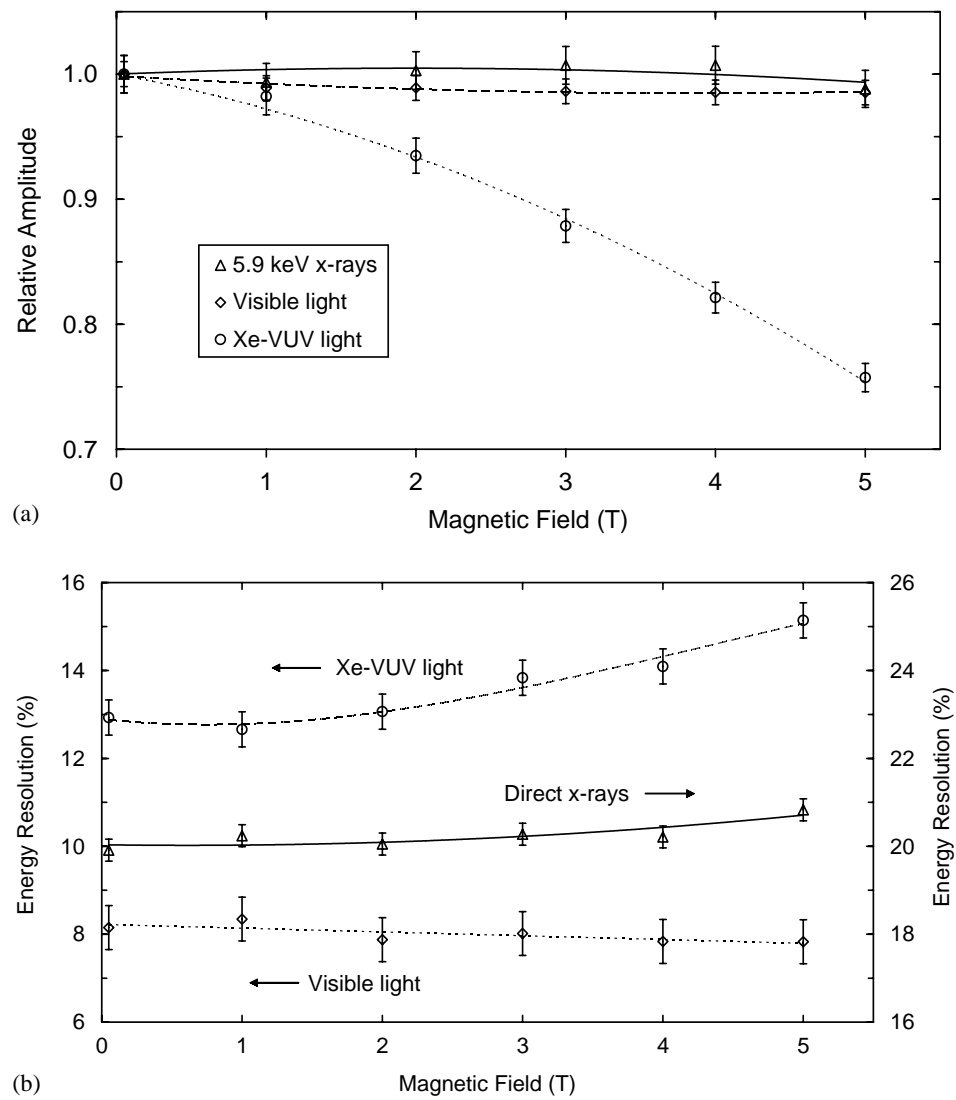

Fig. 5. Relative pulse amplitude (a) and energy resolution (b) for the different detector systems as a function of the magnetic field intensity: direct 5.4-keV X-ray interactions in the LAAPD; visible-light interactions in the LAAPD, resulting from $835 \gamma$-ray interactions in CsI(Tl) and VUV-light interactions in the LAAPD, resulting from 5.9-keV X-ray interactions in a xenon-GPSC.

$24 \%$ at $5 \mathrm{~T}$, while the detector energy resolution degrades from $12.9 \%$ to $15.1 \%$ as the magnetic field increases from 0 to $5 \mathrm{~T}$.

The effect of the magnetic field on the GPSC scintillation is negligible [20,21]: since the X-ray beam is well collimated and aligned with the axis, possible variations in the solid angle subtended by the LAAPD relative to the region where the scintillation takes place, due to Lorentz angle effect [20], are negligible. Thus, the noticeable influence of the magnetic field has its origin in the VUV-light detection in the LAAPD. Although the dependence of the avalanche photodiode amplitude and the respective statistical fluctuations on the magnetic field is not significant for X-ray and visible-light detection, it becomes important for
VUV-detection. Since VUV photons interact within the first atomic layers, the effect of the magnetic field on the photoelectrons and subsequent secondary electrons diffusion may be responsible for increased charge carrier losses to the front electrode with increasing magnetic field. Penetration depths in Si are about $5 \mathrm{~nm}$ and $1 \mu \mathrm{m}$ for $172-$ and $520-\mathrm{nm}$ photons [22], respectively, and $22 \mu \mathrm{m}$ for 5.4-keV X-rays [23].

Presently, the LAAPD manufacturing technology is well established and quite good reproducibility is obtained. Thus, it is expected that the observed behaviour for individual LAAPDs are representative for any of these devices [24,25]. The response to VUV could be improved if the electric field intensity in the drift region would be 
increased. However, this cannot yet be achieved due to the increase of discharge probability at the surface [25].

\section{Conclusions}

We have shown that large-area avalanche photodiodes can operate in strong magnetic fields up to $5 \mathrm{~T}$ with negligible performance degradation when used for X-ray or visible-light detection. However, for VUV-light detection a noticeable degradation in the avalanche photodiode pulseamplitude and in the respective statistical fluctuations is observed. The relative pulse amplitude decreases with increasing magnetic field intensity, reaching a reduction of $24 \%$ at $5 \mathrm{~T}$.

\section{Acknowledgements}

Support is acknowledged to Fundação para a Ciência e a Tecnologia (FCT) through Projects POCTI/FIS/13140/98 and CERN/FIS/43785/01, and to Swiss National Science Foundation. J.F.C.A. Veloso acknowledges support grant from FCT.

\section{References}

[1] E. Lorentz, S. Natkaniec, D. Renker, B. Schwartz, Nucl. Instr. and Meth. A 344 (1994) 64.

[2] R. Farrel, F. Olschner, K. Shah, M.R. Squillante, Nucl. Instr. and Meth. A 387 (1997) 194.

[3] A. Karar, Y. Musienko, J.C. Vanal, Nucl. Instr. and Meth. A 428 (1999) 413.

[4] K. Deiters, Y. Musienko, S. Nicol, B. Patel, D. Renker, S. Reucroft, R. Rusack, T. Sakhelashvili, J. Swain, P. Vikas, Nucl. Instr. and Meth. A 442 (2000) 193.

[5] B. Pichler, G. Böning, E. Lorenz, R. Mirzoyan, W. Pimpl, M. Schwaiger, S.I. Ziegler, IEEE Trans. Nucl. Sci. NS-45 (1998) 1298.

[6] A. Ruru Chen, A. Fremout, S. Tavernier, P. Bruyndonckx, D. Clément, J.-F. Loude, C. Morel, Nucl. Instr. and Meth. A 433 (1999) 637.
[7] A. Ochi, Y. Nishi, T. Tanimori, Nucl. Instr. and Meth. A 378 (1996) 267.

[8] L.M.P. Fernandes, J.A.M. Lopes, J.M.F. dos Santos, C.A.N. Conde, X-ray Spectrom. 30 (2001) 164.

[9] J.A.M. Lopes, J.M.F. dos Santos, R.E. Morgado, C.A.N. Conde, IEEE Trans. Nucl. Sci. NS-48 (2001) 312.

[10] C.M.B. Monteiro, J.A.M. Lopes, J.M.F. dos Santos, C.A.N. Conde, IEEE Trans Nucl. Sci. NS-48 (2001) 1081.

[11] V.N. Solovov, A. Hitachi, V. Chepel, M.I. Lopes, R. Ferreira Marques, A.J.P.L. Policarpo, Nucl. Instr. Meth. A 488 (2002) 572.

[12] F. Kottmann, et al. (24 authors), Hyp. Interact. 138 (2001) 55.

[13] J.F.C.A. Veloso, J.A.M. Lopes, C.A.N. Conde, L.M.P. Fernandes, E.D.C. Freitas, O. Huot, P. Knowles, F. Kottmann, F. Mulhauser, J.M.F. dos Santos, D. Taqqu, IEEE Trans. Nucl. Sci. 49 (2002) 899.

[14] A.Q.R. Baron, S.L. Ruby, Nucl. Instr. Meth. A 343 (1994) 517.

[15] L.M.P. Fernandes, J.A.M. Lopes, C.M.B. Monteiro, J.M.F. dos Santos, R.E. Morgado, Nucl. Instr. Meth. A 478 (2002) 395.

[16] J.A.M. Lopes, L.M.P. Fernandes, J.M.F. dos Santos, R.E. Morgado, C.A.N. Conde, VUV detection in large-area avalanche photodiodes as a function of temperature, presented to 3rd Beaune Conference on New Developments in Photondetection, Beaune, France, June 17-21, 2002, Nucl. Instr. and Meth. A. submitted for publication.

[17] D. Taqqu, Nucl. Instr. Meth. A 247 (1986) 288.

[18] E.M. Gullikson, E. Gramsch, M. Szawlowski, Appl. Opt. 34 (1995) 4662.

[19] J.P. Pansart, Nucl. Instr. and Meth. A 387 (1997) 186.

[20] J.F.C.A. Veloso, J.M.F. dos Santos, C.A.N. Conde, F. Mulhauser, P. Knowles, C. Donche-Gay, O. Huot, D. Taqqu, F. Kottmann, Nucl. Instr. and Meth. A 460 (2001) 297.

[21] J.F.C.A. Veloso, C.A.N. Conde, O. Huot, P. Knowles, F. Kottmann, F. Mulhauser, J.M.F. dos Santos, D. Taqqu, Nucl. Instr. Meth. A 489 (2002) 266.

[22] T.W. Barnard, M.I. Crockett, J.C. Ivaldi, P.L. Lundberg, D.A. Yates, P.A. Levine, D.J. Sauer, Anal. Chem. 65 (1993) 1231-1239.

[23] L.S. Birks, J. Criss, Mass photoelectric absorptions coefficients, In: J. W. Robinson (Ed.), Handbook of Spectroscopy, Vol. III, CRC Press, Boca Raton, FL, USA, 1981.

[24] J.A.M. Lopes, Gas proportional scintillation counters: new applications and technologies in integrated photosensors, Ph.D. Thesis, Physics Department, University of Coimbra, Portugal, 2002.

[25] M. Szawlowsky, Advanced Photonix Inc., private communication. 